\title{
Fatal fungemia by biofilm-producing Trichosporon asahii in a liver transplant candidate
}

\author{
Giusy Tiseo ${ }^{1}$, Roberta Fais ${ }^{2}$, Arianna Forniti ${ }^{1}$, Fabio Melandro $^{3}$, Arianna Tavanti ${ }^{4}$, \\ Emilia Ghelardi ${ }^{2}$, Paolo De Simone ${ }^{3,5}$, Marco Falcone ${ }^{1}$, Antonella Lupetti ${ }^{2}$ \\ ${ }^{1}$ Department of Clinical and Experimental Medicine, University of Pisa, Pisa, Italy; \\ 2Department of Translational Research and of New Technologies in Medicine and Surgery, \\ University of Pisa, Pisa, Italy; \\ ${ }^{3} \mathrm{H}$ epatobiliary surgery and liver transplantation, University of Pisa Medical School Hospital, Pisa, Italy; \\ ${ }^{4}$ Department of Biology, University of Pisa, Pisa, Italy; \\ ${ }^{5}$ Department of Surgical, Medical, Molecular Pathology and Intensive Care, University of Pisa, Pisa, Italy
}

Article received 26 May, 2021; accepted 15 July, 2021

\section{SUMMARY}

Acute-on-chronic liver failure (ACLF) is often associated with a dismal outcome. Infections might preclude access to liver transplantation (LT) for these patients, further reducing their chance of survival. We report the case of a patient with ACLF who died before LT for biofilm-producing Trichosporon asahii fungemia. The patient early started antifungal therapy with anidulafungin, but T. asahii was not susceptible to echinocandins, delaying the start of active antifungal therapy.
Although rare, invasive infections by Trichosporon spp. are associated with high mortality rates due to low antimicrobial susceptibility and production of biofilms on indwelling devices. Early diagnosis and treatment are crucial to reduce mortality and enhance patient survival.

Keywords: Trichosporon, fungal infections, liver transplantation, biofilm, fungi.

\section{INTRODUCTION}

\begin{abstract}
A cute-on-chronic liver failure (ACLF) is associated with high short-term mortality, and the optimum timing and selection criteria for liver transplantation (LT) still remains debated [1]. Patients with ACLF are at high risk of fatal fungal infections, which may increase the risk of wait-list drop-out and mortality [2]. Early diagnosis is therefore crucial in these patients to allow referral to LT.

Trichosporon asahii is responsible for invasive infections and fungemia is the predominant infec-
\end{abstract}

Corresponding author

Marco Falcone

E-mail: marco.falcone@unipi.it tion type, representing two thirds of all invasive infections [3, 4]. Indwelling central venous catheter presence, prior antifungal agent use, haematologic malignancy, and end-stage renal disease are factors associated with invasive T. asahii infection. Mortality rates in these patients is as high as $60 \%$ [3]. The ability of T. asahii to produce biofilms on surfaces is one of the major virulence factors of this organism [5]. Several studies showed that $T$. asahii biofilms produce extracellular protease and may lead to increased resistance to antifungal compounds, which may ultimately lead to clinical treatment failure [6, 7].

The optimal treatment of invasive T. asahii infection remains challenging: resistance to echinocandins, together with the propensity to produce biofilm, complicate the clinical decision of the optimal antifungal regimen in these type of infections [8]. 
Here we describe a patient with ACLF who died of systemic infection caused by a biofilm producing T. asahii strain (formerly known as T. beigelii).

\section{CASE PRESENTATION}

A 31-year-old man with a history of alcohol abuse was admitted to the intensive care unit (ICU) of the Pisa University Hospital (Pisa, Italy) due to liver decompensation with severe jaundice, grade 2 encephalopathy, ascites and diffuse edema. He had been hospitalized in another institution for 3 weeks before being transferred to our hospital where he was treated with steroids and plasmapheresis with no benefit (Maddrey score 63.1; Lille score 0.99). The patient had an indwelling urinary catheter.

Upon admission to our hospital, the patient was evaluated by the LT team and was judged according to the practice guidelines as detailed by the American Association for the Study of Liver Disease [9]. At the time of evaluation, he weighed 54 $\mathrm{kg}$ and was 1.75 meters tall. His BMI was $17.9 \mathrm{~kg} /$ $\mathrm{m}^{2}$. He was diagnosed with liver cirrhosis 2 years before. He presented with abdominal distension, jaundice, confusion and lower extremity edema. Bloodwork showed high aspartate aminotransferase and alanine transaminase levels (443 and $107 \mathrm{mU} / \mathrm{mL}$, respectively), creatinine value 3.5 $\mathrm{mg} / \mathrm{dl}$, high total bilirubin $(15.5 \mathrm{mg} / \mathrm{dl})$, and low albumin $(2.2 \mathrm{mg} / \mathrm{dl})$. The laboratory tests also showed leukocytosis with neutrophilia (32.430/ $\mathrm{mm}^{3} ; \quad 93.1 \%$ neutrophils); thrombocytopenia $\left(17.000 / \mathrm{mm}^{3}\right)$, and increased C-reactive protein (3.39 $\mathrm{mg} / \mathrm{dL})$ and procalcitonin $(3 \mathrm{ng} / \mathrm{mL})$. INR was 5.86. Model for end-stage liver disease-sodium (MELD-Na) score was 40 and his Child-Pugh classification was C. It was recommended to undergo LT as soon as clinically feasible due to poor prognosis.

However, the patient was transferred to the ICU due to critically-ill clinical conditions, hypotension $(80 / 50 \mathrm{mmHg})$ requiring vasopressor therapy and hepato-renal syndrome. Haemodialysis was started. A total-body CT scan showed small bilateral pulmonary infiltrates and left pleural effusion. Empirical antimicrobial therapy with meropenem (500 mg q24h), ceftobiprole (250 mg q12h) and anidulafungin (100 mg q24h) was started. The ascites was sampled $\left(270 / \mathrm{mm}^{3}\right.$ leukocytes) and the culture was negative. About 24 hours after admission, both urine and blood cultures were positive for yeast cells eventually identified as Trichosporon asahii. Blood specimen was inoculated into blood culture bottles (Plus aerobic/F and Plus Anaerobic/F, Becton Dickinson \& Co, BD, Milan, Italy) and transferred to the Bactec FX instrument (Becton Dickinson, Franklin Lakes, NJ, USA) for monitoring microbial growth. Identification of the clinical isolate was obtained by MALDI-TOF mass spectrometry and antimicrobial susceptibility (AST) was obtained with Sensititre $^{\mathrm{TM}}$ YeastOne $^{\mathrm{TM}}$ (Remel Inc., Thermo Fisher Scientific, United Kingdom). NaPB, pH 7). According to the drug susceptibility profile (amphotericin B MIC $1 \mathrm{mcg} / \mathrm{mL}$; anidulafungin MIC 8 $\mathrm{mcg} / \mathrm{mL}$; caspofungin MIC $8 \mathrm{mcg} / \mathrm{mL}$; fluconazole MIC $4 \mathrm{mcg} / \mathrm{mL}$; fluorocytosine MIC $4 \mathrm{mcg} /$ $\mathrm{mL}$; itraconazole MIC $0.5 \mathrm{mcg} / \mathrm{mL}$; micafungin MIC $8 \mathrm{mcg} / \mathrm{mL}$; posaconazole MIC $0.5 \mathrm{mcg} / \mathrm{mL}$; voriconazole $0.25 \mathrm{mcg} / \mathrm{mL}$ ), antifungal therapy was replaced by liposomal amphotericin B (150 $\mathrm{mg}$ q24h). However, the patient clinical status deteriorated even further and he died the following day.

An evaluation of biofilm production was performed: biofilm production was evaluated at 24 and $36 \mathrm{~h}$. Biofilm formation assays were performed as previously described for T. asahii [10]. Briefly, fungal suspensions were prepared at $1 \times 10^{6}$ cells $/ \mathrm{mL}$ in RPMI 1640 medium supplemented with glucose ( $2 \%$ final concentration), buffered with 3-(N-morpholino)-propanesulfonic acid (MOPS; Sigma Aldrich, St. Louis, USA) and transferred in polystyrene, flat-bottomed, 96-well microtiter plates $(100 \mu \mathrm{L} /$ well $)$. After 24 -h incubation at $37^{\circ} \mathrm{C}$, the medium was removed and substituted with fresh $2 \%$ glucose supplemented RPMI medium. At the various incubation times, non-adhered cells were removed by washing twice with phosphate buffered saline (PBS). Biofilm production was evaluated spectrophotometrically by measuring the total biofilm biomass and quantifying cellular metabolic activity by the XTT/menadione assay. XTT (2,3-Bis-(2-Methoxy4-Nitro-5-Sulfophenyl)-2H-Tetrazolium-5-Carboxanilide, Euroclone SpA, Italy) solution was prepared at $0.5 \mathrm{mg} / \mathrm{mL}$ in PBS buffer and mixed with a menadione solution dissolved in acetone at a final concentration of $1 \mu \mathrm{M}$. An aliquot of $100 \mu \mathrm{L}$ $\mathrm{XTT} /$ menadione solution was inoculated into each well of the plate and incubated for $2 \mathrm{~h}$ in the 
dark at $37^{\circ} \mathrm{C}$. Next, the supernatant $(80 \mu \mathrm{L})$ was transferred into a new 96-well microtiter plate to measure colorimetric changes at $490 \mathrm{~nm}\left(\mathrm{OD}_{\lambda 490 \mathrm{~nm}}\right)$ using an automated plate reader (Multiskan ${ }^{\mathrm{TM}} \mathrm{FC}$ Microplate Photometer, Thermo Scientific). Background optical density was subtracted from the values measured in each well. Three independent experiments were performed, each in triplicate. Results about the biofilm-forming ability after 24 and 36-hour incubation is shown in Figure 1. No significant difference was observed between time points. T. asahii was classified as moderate biofilm producer.

The ability of T. asahii to produce biofilm was further investigated using an in vitro model of catheter-associated biofilm formation. Peripheral Teflon catheters (PVC) of $1 \mathrm{~mm}$ diameter and $32 \mathrm{~mm}$ length (Deltaven FEP, Deltamed SpA; Mantova, Italy) were inoculated with $20 \mu \mathrm{L}$ of yeast suspension and then placed into a micro-centrifuge tube with $1 \mathrm{~mL}$ of RPMI. After 6, 12, 24, and 36-hour

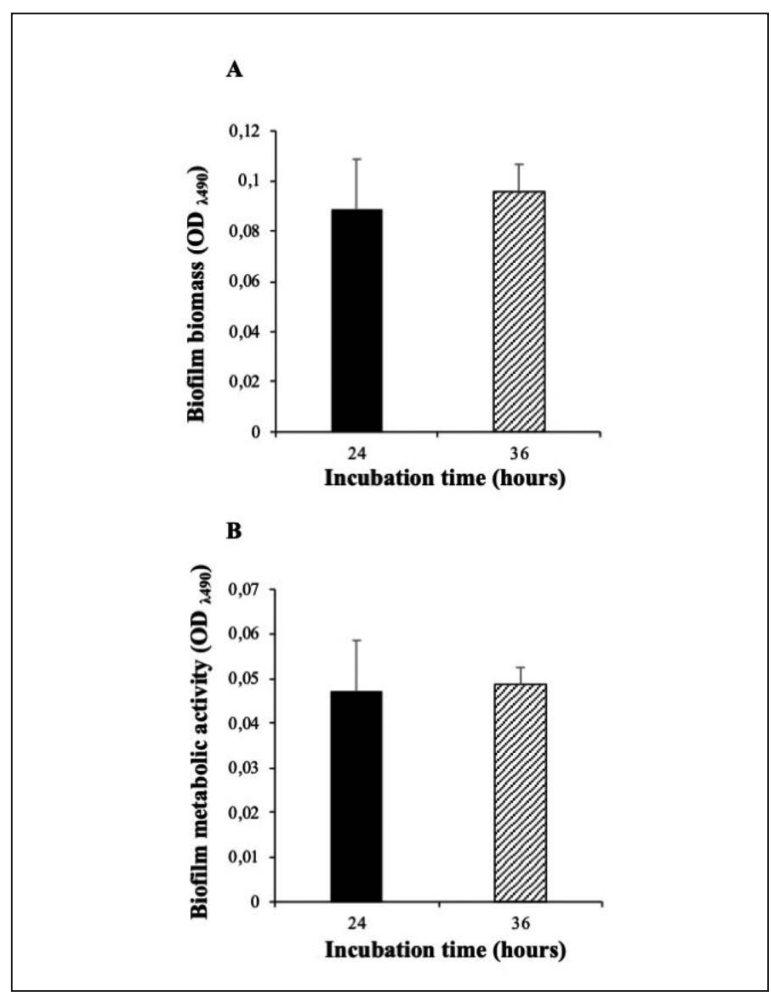

Figure 1 - T. asahii biofilm biomass (A) and metabolic activity (B) after incubation in $2 \%$ glucose-supplemented RPMI for 24 and 36 hours.

Data are expressed as means of three independent experiments \pm SD

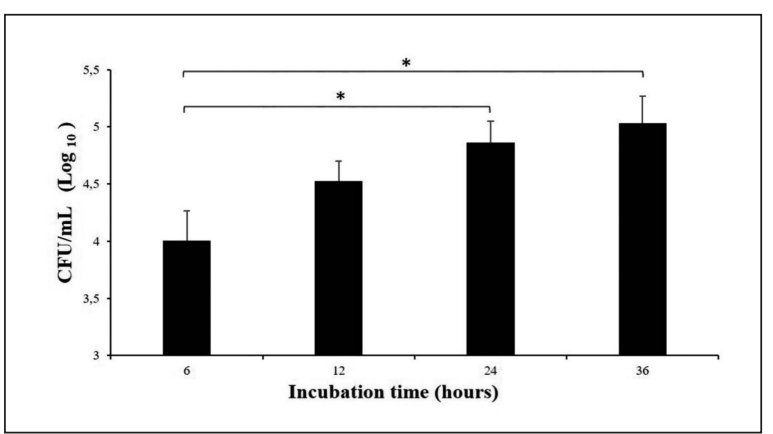

Figure 2 - Biofilm embedded $T$. asahii cells in the catheter lumen.

Catheters (PVC) were inoculated with T. asahii (106 cells $/ \mathrm{mL}$ ) and incubated in $2 \%$ glucose supplemented RPMI for $6,12,24$, and $36 \mathrm{~h}$ at $37^{\circ} \mathrm{C}$. Biofilm embedded cells were detached from the catheter lumen by sonication and the number of cells was determined microbiologically. Data are expressed as means of three independent experiments $\pm \mathrm{SD}$. ${ }^{*} \mathrm{P} \leq 0.01$.

incubation at $37^{\circ} \mathrm{C}$, the colonized catheters were washed in PBS. Next, they were sonicated for 5 min and vortexed for further $2 \mathrm{~min}$, to allow complete detachment of biofilm embedded cells from the catheter lumen. The ability of T. asahii to form biofilm in the in vitro catheter model was evaluated after 6, 12, 24 and 36-hour incubation at $37^{\circ} \mathrm{C}$. Data obtained from colonized catheters were expressed as number of cells (CFU/mL) and showed a significant $(p<0.01)$ difference between 6 and 24hour incubation time points as well as between 6 and 36 hours (Figure 2).

\section{DISCUSSION}

The present case highlights the challenges in the management of ACLF patients and their rapid clinical deterioration. In this setting, timing of LT - the so-called transplant window - is narrow and infections may increase the risk of wait-list dropout and mortality. Early diagnosis and treatment of infections is crucial in these patients, since infections may jeopardize access to LT.

The emergence of less common, albeit clinically relevant, fungal pathogens including rare yeasts, contributes to the high morbidity and mortality rates observed in immunosuppressed patients. $T$. asahii life-threatening invasive infections generally occur in immunosuppressed hosts and in critically ill patients [11]. The deteriorated clinical conditions and the length of hospital stay prior to transfer to our center might have been predisposing factors for T. asahii bloodstream infection in 
our patient. Moreover, the presence of an indwelling urinary catheter may have facilitated yeast colonization of the urinary tract mucosa and subsequent spread to the bloodstream. This hypothesis is further supported by the in vitro results, showing the T. asahii strain ability to produce biofilm on abiotic surfaces. This feature may represent a relevant virulence factor, reducing drug penetration and effectiveness [11]. Remarkably, we reproduced an in vitro model of catheter-associated biofilm formation by T. asahii and observed a significant difference between different incubation time points, highlighting the ability of this yeast to increase the biofilm production over time. Accordingly to our finding, Cordeiro and colleagues demonstrated that T. asahii produce persister cells in their biofilms that may provide an inoculum for cell growth and new biofilm formation [6]. Persister cells, a subpopulation of dormant cells within biofilms, may be responsible for relapsing infections, as they tolerate antifungal exposure and can revert to an actively growing state, repopulating the biofilm, once treatment is ceased [6].

This characteristic may be important from a clinical point of view:

1) the increased biofilm formation over time may lead to a reduced activity of antifungal drugs even if active antifungal treatment is early started;

2) it may justify the difficulty in the eradication of the organism, especially when source control is not promptly performed or in patients who are catheter dependent or have risks associated with catheter removal.

The biofilm formation and persistence significantly contribute to the reduced susceptibility of $T$. asahii to antifungal agents. Trichosporon spp. are characterized by a low susceptibility to commonly used antifungal drugs. Although many case reports describe the use of amphotericin B (AMB) for the treatment of trichosporonosis, in vitro resistance to AMB has been detected in a number of isolates collected from neutropenic patients with disseminated infection [12, 13]. In our case, we used anidulafungin as first empirical treatment choice, because it is active against the majority of yeasts and is not metabolized by the liver and no dose adjustments are required for patients with hepatic dysfunction. However, anidulafungin is not active in vitro against Trichosporon spp and the delay in starting an appropriate antifungal treatment might have contributed to the fatal outcome $[14,15]$. Considering also the synergistic activity against biofilm, some authors suggest the use of voriconazole-amphotericin B combination as a potential therapeutic option for T. asahii fungemia, especially for patients who are catheter dependent or have risks associated with catheter removal [16]. Isavuconazole showed in vitro activity against rare yeast species, including Trichosporon, Saccharomyces, Geotrichum, Pichia and Rhodotorula species, and might be a treatment option in immunosuppressed patients [17]. Further clinical studies are needed for ACLF patients.

In conclusion, early diagnosis and treatment are mandatory in the presence of infections in ACLF patients to increase their access to LT. Based on patient's risk factors and in specific patients' population (including ACLF patients), rare yeasts, like Trichosporon spp. should be considered when initiating an empirical antimicrobial therapy.

\section{Ethics statement}

The study was notified to and approved by the local ethical committee (Comitato Etico di Area Vasta Nord-Ovest) and conducted in full accordance with the principles of the Declaration of Helsinki. Samples were taken as part of the standard patient care and anonymized by the clinical personnel. Research personnel received and used the sample anonymously.

\section{Conflict of interest}

None to declare.

\section{Funding}

This research was supported by the PRIN 2017 grant prot. 20177J5Y3P from the Italian Ministry of Education, University and Research (MIUR).

\section{Acknowledgements}

None

\section{Authors contributions}

$\mathrm{MF}$, AL conceived and designed the experiments; RF performed the experiments; AT, EG, AL, RF analyzed the data; GT drafted the manuscript with the contribution of AF, FM, PDS. All of the authors reviewed and revised the first and final drafts of this manuscript. FrM critically revised the manuscript. 


\section{REFERENCES}

[1] Fernández J, Saliba F. Liver transplantation in patients with ACLF and multiple organ failure: Time for priority after initial stabilization. J Hepatol. 2018; 69 (5), 1004-06.

[2] Sundaram V, Shah P, Wong RJ, et al. Patients with acute on chronic liver failure grade 3 have greater 14day waitlist mortality than status-1a patients. Hepatology. 2019; 70 (1), 334-45.

[3] Kim J, Kim MJ, Chong YP, et al. Comparison of the characteristics of patients with invasive infections and noninvasive infections caused by Trichosporon asahii. Med Mycol. 2021; 59 (3), 296-300.

[4] Li H, Guo M, Wang C, et al. Epidemiological study of Trichosporon asahii infections over the past 23 years. Epidemiol Infect. 2020; 148, e169.

[5] Kurakado S, Miyashita T, Chiba R, Sato C, Matsumoto Y, Sugita T. Role of arthroconidia in biofilm formation by Trichosporon asahii. Mycoses. 2021; 64 (1), $42-7$. [6] Cordeiro RA, Aguiar ALR, da Silva BN, et al. Trichosporon asahii and Trichosporon inkin biofilms produce antifungal-tolerant persister cells. Front Cell Infect Microbiol. 2021; 11, 645812 .

[7] Cordeiro, R, Serpa R, Alexandre CFU, et al. Trichosporon inkin biofilms produce extracellular proteases and exhibit resistance to antifungals. J Med Microbiol. 2015; 64 (11), 1277-86.

[8] Guo LN, Yu SY, Hsueh PR, et al. invasive infections due to Trichosporon: species distribution, genotyping, and antifungal susceptibilities from a multicenter study in China. J Clin Microbiol. 2019; 57, e01505-18.

[9] Martin P, DiMartini A, Feng S, Brown R, Fallon M. Evaluation for liver transplantation in adults: 2013 practice guideline by the American Association for the Study of Liver Diseases and the American Society of Transplantation. Hepatology. 2014; 59 (3), 1144-65.
[10] de Aguiar Cordeiro R, Galdino Pereira LM, Kleybson de Sousa J, et al. Farnesol inhibits planktonic cells and antifungal-tolerant biofilms of Trichosporon asahii and Trichosporon inkin. Medical Mycol. 2019; 57 (8), 1038-45.

[11] de Almeida Júnior JN, Hennequin C. Invasive Trichosporon infection: a systematic review on a re-emerging fungal pathogen. Front Microbiol. 2016; 7, 1629.

[12] Arendrup MC, Boekhout T, Akova M, et al. ESCMID and ECMM joint clinical guidelines for the diagnosis and management of rare invasive yeast infections. Clin Microbiol Infect. 2014; 20 (Suppl. 3), 76-98.

[13] Itoh K, Iwasaki H, Negoro E, et al. Successful treatment of breakthrough Trichosporon asahii fungemia by the combination therapy of fluconazole and liposomal amphotericin b in a patient with follicular lymphoma. Mycopathologia. 2021; 186 (1), 113-17.

[14] Falcone M, Tiseo G, Gutiérrez-Gutiérrez B, et al. GISA (Italian Group for Antimicrobial Stewardship). Impact of initial antifungal therapy on the outcome of patients with candidemia and septic shock admitted to medical wards: a propensity score-adjusted analysis. Open Forum Infect Dis. 2019; 6 (7), ofz251.

[15] Francisco EC, de Almeida Junior JN, Queiroz-Telles F, et al. Correlation of Trichosporon asahii genotypes with anatomical sites and antifungal susceptibility profiles: data analyses from 284 isolates collected in the last 22 years across 24 medical centers. Antimicrob Agents Chemother. 2021; 65 (3), e01104-20.

[16] Liao Y, Yang S, Cong L, Lu X, Ao J, Yang R. In vitro activities of antifungal combinations against biofilms and planktonic forms of clinical Trichosporon asahii isolates. Antimicrob Agents Chemother. 2014; 58 (12), 7615-6. [17] Thompson GR, Wiederhold NP, Sutton DA, Fothergill A, Patterson TF. In vitro activity of isavuconazole against Trichosporon, Rhodotorula, Geotrichum, Saccharomyces and Pichia species. J Antimicrob Chemother. 2009; 64 (1), 79-83. 